\title{
The Dopaminergic and Acetylcholinergic Supplements to Freudian Structural Theory of Psychoanalysis
}

\author{
Zi-Jian Cai \\ CaiFortune TriL Consulting, Suzhou, China \\ Email: hrsh8@126.com
}

How to cite this paper: Cai, Z.-J. (2021) The Dopaminergic and Acetylcholinergic Supplements to Freudian Structural Theory of Psychoanalysis. Open Access Library Journal, 8: e7603.

https://doi.org/10.4236/oalib.1107603

Received: June 1, 2021

Accepted: June 28, 2021

Published: July 1, 2021

Copyright $\odot 2021$ by author(s) and Open Access Library Inc.

This work is licensed under the Creative Commons Attribution International License (CC BY 4.0).

http://creativecommons.org/licenses/by/4.0/

\section{(c) (i) Open Access}

\begin{abstract}
For the Freudian topographic theory of psychoanalysis, it has recently been supplemented the reticular noradrenergic (NA), serotonergic (5-HT) and acetylcholinergic $(\mathrm{ACh})$ systems to demonstrate the differential regulation of unconscious/preconscious/conscious in waking and sleep, applicable to depression and anxiety. For the Freudian structural theory of id/ego/super-ego, it is keen to depict the personal social psychology with selfishness, applicable to schizophrenia. Herein, it is analogously added the neglected reticular dopaminergic (DA) and ACh systems to regulate the id, ego, and super-ego, corresponding to the bodily instincts including motivation, selfish behaviors including skills, and social knowledge including law, respectively. For DA, predominantly it is selective on super-ego, excitatory to id and beneficial to ego; while for $\mathrm{ACh}$, predominantly it is excitatory to super-ego, depressive to id, and restrictive to ego. It is the hyperactivation of DA and downregulation of ACh that results in schizophrenia and dementia, respectively. It is accordingly reconciled the structural theory of psychoanalysis with the present pathological and pharmacological achievements.
\end{abstract}

\section{Subject Areas}

Neuroscience, Psychiatry \& Psychology, Neurology, Psychology

\section{Keywords}

Psychoanalysis, Selfish Behavior, Schizophrenia, Dopamine, Acetylcholine,

Dementia

\section{Introduction}

The Freudian psychoanalysis has become one of the most predominant theoret- 
ical systems in psychology, early, clear and systematic, especially on the interactions and conflicts among the unconscious/preconscious/conscious in mind as topographic theory or id/ego/super-ego as structural theory [1] [2]. The interactions and conflicts of the three mental elements in the topographic theory and structural theory represent the core contents of the Freudian systematic theories in psychoanalysis [1] [2] [3] [4]. The neuropsychoanalysis, as the neurobiological basis of psychoanalysis, is now a prospective field for updated neurobiological integration and investigation [3]-[10].

Recently, while supporting and supplementing the neurobiological interactions and conflicts of unconscious/preconscious/conscious of topographic theory in waking, slow wave sleep (SWS) and rapid eye-movement (REM) sleep for depression and anxiety, Cai additionally supplemented the reticular noradrenergic (NA), serotonergic (5-HT) and acetylcholinergic (ACh) systems to demonstrate the differentiation in interactions and conflicts of emotion (unconscious), memory (preconscious), and conscious during waking, SWS and REM sleep [3], with the SWS and REM sleep manifesting opposite with each other [3] [4] [11] [12]. Earlier, Hobson as well depicted the influence of NA, 5-HT and ACh on waking, SWS and REM sleep, resulting in the different manifestations of emotion, memory and consciousness in these brain states, while with less consideration on the detailed differentiation of their interactions and conflicts in the states [5].

On the other hand, the recent efforts on the neurobiological id/ego/super-ego of structural theory have neglected the roles of ascending reticular systems [6] [8] [9], incomprehensive in consideration of brain structures involved. In this article, it is supplemented the ascending reticular systems to the Freudian structural theory of psychoanalysis.

\section{Method}

To deal with such an important but big topic, there is no better and more convincing method than integrative reviewing all relevant fields of studies. Citing updated relevant reviews or, if not available, salient and repeated experimental results in subfields are the best method. It is necessary to clarify that the presently widely utilized meta-analysis fits investigation of a specific topic in a well-studied subfield, but not for integration or summarization from several fields like this paper.

Papers were searched out from Pubmed and Baidu Xueshu. The updated relevant reviews in subfields were given priority to cite. If not available, relevant reviews were cited. If still unavailable, the salient and repeated experimental results of original articles in subfields were cited. The papers written by the author were cited with priority above all of these so as to demonstrate the expertise of the author to write this article.

\section{Results}

\subsection{The Contemporary Neurobiological Studies of Ego}

Structural theory divides the mental elements for personal social psychology into 
the id, ego, and super-ego [1] [2]. The definitions of id, ego, and super-ego may be a little different in different situations according to the needs of use. For the convenience of use in neurobiology while in consistence with other neurobiological terms, the id is defined as the basic instincts including motivation [9], unorganized and unconscious, operating without realism or foresight [1] [2] [9] [10]. The ego develops slowly and gradually, being concerned with selfish behaviors including skills mediating between the urging of the id and the realities of the external world [1] [2] [6] [8], and thus operating on reality. The super-ego is held to be the self-observation, self-criticism and other reflective behaviors or common knowledge including law [1] [2]. Obviously, even though called as structural theory of psychoanalysis, the neurobiological counterparts of id/ego/super-ego are all behavioral or psychological. Table 1 outlines the neurobiological counterparts of id/ego/super-ego.

The most noteworthy trends in recent progressions for neurobiology of structural theory of psychoanalysis have pointed to the underlying brain structures and circuits responsible for the id and ego [6] [8] [9]. With regard to id, some authors suggested that the motivation could be more appropriate to represent id than drive [9], while others pointed to the activation of ascending dopaminergic (DA) systems by pleasure [10]. With regard to ego, some authors suggested that the cortical midline structures (CMS) and the default mode network (DMN) correspond to the neural correlates of selfish ego in schizophrenia [6], while others suggested that the cortical parieto-frontal circuits and DMN correspond to the conscious and unconscious part of ego, respectively [8].

However, these neurobiological progressions are far from satisfactory to demonstrate the structural theory of psychoanalysis. 1) Very few neurobiological progressions have been made related to the super-ego of structural theory. 2) Even though the CMS, DMN and cortical parieto-frontal circuits were suggested to be related to ego [6] [8], it has been demonstrated that their specificity was not high sufficiently [6]. Especially, it is difficult to separate the super-ego or even id away from the brain structures or circuits of ego, which results in difficulty for neurobiology to clarify the interactions and conflicts of id, ego, and super-ego with them. 3) Most of the neurobiological progressions on structural theory have neglected the ascending reticular systems [6] [8] [9], therefore incomprehensive in consideration of related brain structures or circuits. Even though some authors have pointed to the ascending DA systems as related to pleasure [10], they are too few and lack of considering the ego and super-ego.

Table 1. The neurobiological counterparts of id/ego/super-ego.

\begin{tabular}{cc}
\hline Mental Element & Neurobiological Counterparts \\
\hline Id & The basic instincts including motivation \\
Ego & The selfish behaviors including skills \\
Super-ego & The reflective behaviors or common knowledge including law \\
\hline
\end{tabular}


In contrast, for the topographic theory on depression and anxiety, Cai added the reticular NA, 5-HT and ACh systems to demonstrate the differentiated interactions and conflicts of emotion (unconscious), memory (preconscious), and conscious in waking, SWS and REM sleep, respectively [3], in which the SWS and REM sleep manifested opposite with each other [3] [4] [11] [12], quite comprehensive and detailed in mechanisms.

Accordingly, it is obviously necessary to extend the neurobiological consideration of structural theory of psychoanalysis to more brain structures or circuits, especially including the neglected ascending reticular systems.

\subsection{The Ascending DA Regulation of Id, Ego and Super-Ego}

The structural theory of psychoanalysis is advantageous in that it depicts the personal social psychology by the interactions and conflicts of id, ego and super-ego, emphasizing the importance of selfishness and its interaction with reality, therefore applicable to schizophrenia [6]. Accordingly, it is herein first adopted the schizophrenia-related ascending DA systems to inspect their regulation on id, ego and super-ego.

With regard to id including motivation, the DA systems are excitatory. As mentioned above, some authors have suggested that the ascending DA should be related to pleasure [10]. Besides, the ascending DA systems have directly been depicted as dopamine motive system [13]. In further, it has been demonstrated that the DA in both ventral tegmental area and substantia nigra could contribute to the regulation of motivation [14]. It was noted that the DA systems innervated and regulated the GABAergic interneurons in the basal ganglia, and could directly disinhibit the motivation [14].

With regard to ego representing selfish behaviors including skills, DA can exert the facilitatory effects as manifested in schizophrenia and procedural memory. On the one hand, if hyperactivation of DA may excessively facilitate the ego, it may thus result in psychotic disease like schizophrenia. Indeed, modern technologies have now been able to demonstrate in consensus that the DA in associative striatum is increased in schizophrenia [15] [16], while dysfunction of DA in other brain regions of schizophrenia remains controversial [16]. On the other hand, acquisition of procedural memory can improve the ability of ego as selfish behaviors and skills. Many lines of evidence have demonstrated that the DA of basal ganglia is required for various procedural learning and memory [17] [18], while some authors have even considered the impairment on some types of procedural memories in Parkinson's disease as important for classification of procedural memories as memory impairment in hippocampal amnesia [18].

With regard to super-ego representing the many reflective behaviors or common knowledge including law, DA can coordinate the selective attention based on self need. It has been reviewed that both prefrontal cortex and DA are involved in selective attention and working memory [19]. Besides, in the attention-deficit/hyperactivity disorder, DA dysfunction has been considered as 
one of the important pathological causes [20].

It is necessary to point out that the ascending DA systems from substantia nigra and ventral tegmental area may exert different roles [16]. Accordingly, the effects of DA on id/ego/super-ego of structural theory can only be considered as predominant or gross from both of substantia nigra and ventral tegmental area. Detailed depiction of their effects requires further investigation.

\subsection{The Ascending ACh Regulation of Id, Ego and Super-Ego}

Besides the ascending DA systems, the dementia-related ascending ACh systems are herein also adopted to inspect their regulation on id, ego and super-ego.

With regard to id including motivation, the ACh systems are depressive. Hyper- and hypo-cholinergic states may result in depression and mania respectively [21]. Correspondingly, $\mathrm{ACh}$ is mainly excitatory to the limbic activities [22].

With regard to ego as selfish behaviors and skills, ACh can exert some restrictive roles. In the famous working memory consolidation model of Mishkin and Appenzeller, lesions of the ascending ACh systems impaired biochemical consolidation of working memories [23], helping restrict the behaviors by avoidance of failures and improvement of success. Besides, some authors have suggested that downregulation of ACh activity may be relevant to some schizophrenia [24], opposite to that of DA.

With regard to super-ego for reflective behaviors or common knowledge and law, ACh mainly helps activation of them including excitation of relevant words in sentence [25] [26], recall of useful declarative memories [27] [28] [29], pharmacological therapies of dementia, and so on. Gamma bands are subject to modulation by the ascending cholinergic systems [30] [31]. The gamma bands of high frequency have been shown to be associated with the word congruency at sentential level [25] [26]. Likewise, recall of recent declarative memories has as well been shown to be associated with the theta-gamma coupling [27] [28] [29]. On the other hand, it has recently been reviewed that the ACh systems are involved in the pathophysiology and treatment of Alzheimer's dementia [32].

It is as well necessary to note that the ascending ACh systems may activate various subtypes of receptors. In this regard, the above mentioned effects of ACh on id/ego/super-ego should be considered as predominant or gross. It would require more investigations for depiction of their details.

\section{Discussions and Perspectives}

The id/ego/super-ego mental elements of Freudian structural theory of psychoanalysis correspond in neurobiology to the basic instincts including motivation [1] [2] [9] [10], selfish behaviors including skills [1] [2] [6] [8], and reflective behaviors or common knowledge including law [1] [2], respectively. However, they have been vague and mixed with each other in neurobiological structures up to now. Enlightened by the success of addition of reticular NA, 5-HT and ACh systems to account for the differentiation in interactions and conflicts 
of emotion (unconscious), memory (preconscious), and conscious during waking, SWS and REM sleep [3], which supports and supplements the Freudian topographic theory, in this article it is analogously supplemented the neglected reticular DA and ACh systems as regulation onto the id/ego/super-ego of structural theory.

As results, it is summarized that the DA systems are excitatory to the motivation of id [10] [13] [14], beneficial to ego [15] [16] including procedural memory [17] [18], and selective to super-ego on attention [19] [20], while the ACh systems are depressive to the motivation of id [21] [22], restrictive to ego including procedural memory [23] [24], and excitatory to super-ego [25] [26] [27] [28] [29] [32]. The effects of DA and ACh systems considered herein are both manifested as predominant or gross, with details requiring more investigations. Table 2 outlines these new effects of regulations.

These interpretations, supplemented by the regulation of DA and ACh systems onto id/ego/super-ego, are indispensably useful to scientifically clarify the interactions and conflicts of id/ego/super-ego elements of Freudian structural theory of psychoanalysis. Besides, with such supplementation by DA and ACh, the Freudian structural theory of psychoanalysis can further be reconciled well with the present pathological and pharmacological achievements related to DA and ACh on schizophrenia [15] [16] and dementia [32], respectively.

As psychoanalysis emphasizes revealing the underlying mental processes of patients, the structural theory of psychoanalysis supplemented by DA and ACh regulations would be more accurate and scientific than the modern behavioral approaches and therapies, thus would be improved, and be prospective to reoccupy the predominant role in psychotic diagnosis and therapy in future. Especially in the small psychotic clinics or psychological consultants without complex equipments as in big hospitals, because of its advantage as clear and systematic theory easy for applications, the DA/ACh supplemented structural theory of psychoanalysis would be more useful than any other psychological methods or tools available at present on psychotic diagnosis and therapy for schizophrenia and dementia.

It is also necessary to point out that the DA/ACh supplemented structural theory of Freudian psychoanalysis is herein considered along with motivation, selfishness, schizophrenia, dementia, and so on. Whether and how they can be applied to other psychotic diseases such as depression or anxiety would require

Table 2. The DA and ACh regulation of id/ego/super-ego.

\begin{tabular}{ccc}
\hline Mental Element & DA & ACh \\
\hline Id & Excitatory to motivation & Depressive to motivation \\
Ego & $\begin{array}{c}\text { Beneficial to ego including } \\
\text { procedural memory }\end{array}$ & $\begin{array}{c}\text { Restrictive to ego including } \\
\text { procedural memory }\end{array}$ \\
Super-ego & Selective attention & Excitatory \\
\hline
\end{tabular}


further investigation. Notably, based on depression and anxiety, Cai has supported and supplemented the differentiated neurobiological interactions and conflicts of unconscious/preconscious/conscious in topographic theory of Freudian psychoanalysis in waking, SWS and REM sleep, by addition of the reticular NA, 5-HT and ACh systems [3] [4].

It is further necessary to remind that, besides the ascending DA and ACh systems, the reticular NA, 5-HT systems may also regulate the id/ego/super-ego of Freudian structural theory, which requires further investigation.

\section{Conclusion}

The id/ego/super-ego of Freudian structural theory corresponds to the neurobiological bodily instincts including motivation, selfish behaviors including skills, and social knowledge including law, respectively. In this article, it is supplemented the neglected ascending reticular DA and ACh systems to regulate the id, ego, and super-ego. It is summarized that the DA systems are excitatory to the motivation of id, beneficial to ego including procedural memory, and selective to super-ego on attention, while the ACh systems are depressive to the motivation of id, restrictive to ego including procedural memory, and excitatory to super-ego. It is considered these effects of DA and ACh systems as predominant or gross, with details requiring further investigations. Hyperactivation of DA and decrement of ACh would result in schizophrenia and dementia, respectively. Accordingly, the achievements herein include well the present pathological and pharmacological progressions related to DA and ACh on respective schizophrenia and dementia, while can further be more accurate and scientific to reveal the underlying mental processes via Freudian psychoanalytic interactions.

\section{Acknowledgements}

It is herein acknowledged that MingXun Cai paid the Open Access publication fee of this paper.

\section{Conflicts of Interest}

The author declares no conflicts of interest regarding the publication of this paper.

\section{References}

[1] Psychoanalysis (2021) Wikipedia, The Free Encyclopedia. https://en.wikipedia.org/wiki/psychoanalysis

[2] Bergmann, M.S. (2008) The Mind: Psychoanalytic Understanding Then and Now. Psychoanalytic Quarterly, 77, 139-166. https://doi.org/10.1002/j.2167-4086.2008.tb00337.x

[3] Cai, Z.-J. (2016) Progressions of Sleep, Memory and Depression Applicable to Psychoanalysis: A Review. Current Psychiatry Reviews, 12, 240-245. https://doi.org/10.2174/1573400512666160610083505

[4] Cai, Z.-J. (2018) Evolution of Psychoanalytic Interactions and Conflicts in Verte- 
brates. Sleep and Hypnosis, 20, 1-7. https://doi.org/10.5350/Sleep.Hypn.2016.18.0128

[5] Hobson, J.A. (1999) The New Neuropsychology of Sleep: Implications for Psychoanalysis. Neuropsychoanalysis, 1, 157-183. https://doi.org/10.1080/15294145.1999.10773258

[6] Salone, A., Di Giacinto, A., Lai, C., De Berardis, D., Iasevoli, F., Fornaro, M., De Risio, L., Santacroce, R., Martinotti, G. and Giannantonio, M.D. (2016) The Interface between Neuroscience and Neuro-Psychoanalysis: Focus on Brain Connectivity. Frontiers in Human Neuroscience, 10, Article No. 20. https://doi.org/10.3389/fnhum.2016.00020

[7] Johnson, B. and Flores Mosri, D. (2016) The Neuropsychoanalytic Approach: Using Neuroscience as the Basic Science of Psychoanalysis. Frontiers in Psychology, 7, Article No. 1459. https://doi.org/10.3389/fpsyg.2016.01459

[8] Rizzolatti, G., Semi, A.A. and Fabbri-Destro, M. (2014) Linking Psychoanalysis with Neuroscience: The Concept of Ego. Neuropsychologia, 55, 143-148. https://doi.org/10.1016/j.neuropsychologia.2013.10.003

[9] Meissner, W.W. (2009) The Question of Drive vs. Motive in Psychoanalysis: A Modest Proposal. Journal of the American Psychoanalytic Association, 57, 807-845. https://doi.org/10.1177\%2F0003065109342572

[10] Chenu, A. and Tassin, J.-P. (2014) Pleasure: Neurobiological Conception and Freudian Conception. L'Encéphale, 40, 100-107. https://doi.org/10.1016/j.encep.2013.06.003

[11] Cai, Z.-J. (1991) The Functions of Sleep: Further Analysis. Physiology \& Behavior, 50, 53-60. https://doi.org/10.1016/0031-9384(91)90497-C

[12] Cai, Z.-J. (1995) An Integrative Analysis to Sleep Functions. Behavioural Brain Research, 69, 187-194. https://doi.org/10.1016/0166-4328(95)00005-E

[13] Volkow, N.D., Wise, R.A. and Baler, R. (2017) The Dopamine Motive System: Implications for Drug and Food Addiction. Nature Reviews Neuroscience, 18, 741-752. https://doi.org/10.1038/nrn.2017.130

[14] Ikemoto, S., Yang, C. and Tan, A. (2015) Basal Ganglia Circuit Loops, Dopamine and Motivation: A Review and Enquiry. Behavioural Brain Research, 290, 17-31. https://doi.org/10.1016/j.bbr.2015.04.018

[15] Kesby, J.P., Eyles, D.W., McGrath, J.J. and Scott, J.G. (2018) Dopamine, Psychosis and Schizophrenia: The Widening Gap between Basic and Clinical Neuroscience. Translational Psychiatry, 8, Article No. 30. https://doi.org/10.1038/s41398-017-0071-9

[16] Weinstein, J.J., Chohan, M.O., Slifstein, M., Kegeles, L.S., Moore, H. and Abi-Dargham, A. (2017) Pathway-Specific Dopamine Abnormalities in Schizophrenia. Biological Psychiatry, 81, 31-42. https://doi.org/10.1016/j.biopsych.2016.03.2104

[17] Shohamy, D., Myers, C.E., Kalanithi, J. and Gluck, M.A. (2008) Basal Ganglia and Dopamine Contributions to Probabilistic Category Learning. Neuroscience and Biobehavioral Reviews, 32, 219-236. https://doi.org/10.1016/j.neubiorev.2007.07.008

[18] Foerde, K. and Shohamy, D. (2011) The Role of the Basal Ganglia in Learning and Memory: Insight from Parkinson's Disease. Neurobiology of Learning and Memory, 96, 624-636. https://doi.org/10.1016/j.nlm.2011.08.006

[19] Bahmani, Z., Clark, K., Merrikhi, Y., Mueller, A., Pettine, W., Vanegas, M.I., Moore, T. and Noudoost, B. (2019) Prefrontal Contributions to Attention and Working Memory. Current Topics in Behavioral Neurosciences, 41, 129-153. 
https://doi.org/10.1007/7854 $2018 \quad 74$

[20] Genro, J.P., Kieling, C., Rohde, L.A. and Hutz, M.H. (2010) Attention-Deficit or Hyperactivity Disorder and the Dopaminergic Hypotheses. Expert Review of Neurotherapeutics, 10, 587-601. https://doi.org/10.1586/ern.10.17

[21] Dilsaver, S.C. (1986) Cholinergic Mechanisms in Depression. Brain Research Reviews, 11, 285-316. https://doi.org/10.1016/0165-0173(86)90016-0

[22] Fisahn, A., Pike, F.G., Buhl, E.H. and Paulsen, O. (1998) Cholinergic Induction of Network Oscillations at $40 \mathrm{~Hz}$ in the Hippocampus in Vitro. Nature, 394, 186-189. https://doi.org/10.1038/28179

[23] Mishkin, M. and Appenzeller, T. (1987) The Anatomy of Memory. Scientific American, 256, 80-89. https://doi.org/10.1038/scientificamerican0687-80

[24] Tani, M., Akashi, N., Hori, K., Konishi, K., Kitajima, Y., Tomioka, H., Inamoto, A., Hirata, A., Tomita, A., Koganemaru, T., Takahashi, A. and Hachisu, M. (2015) Anticholinergic Activity and Schizophrenia. Neurodegenerative Diseases, 15, 168-174. https://doi.org/10.1159/000381523

[25] Cai, Z.-J. (2017) The Neurobiological Models of Language: An Updated Review. Psychology \& Neuroscience, 10, 297-306. https://doi.apa.org/doi/10.1037/pne0000101

[26] Lewis, A.G. and Bastiaansen, M. (2015) A Predictive Coding Framework for Rapid Neural Dynamics during Sentence-Level Language Comprehension. Cortex, 68, 155-168. https://doi.org/10.1016/j.cortex.2015.02.014

[27] Cai, Z.-J. (2018) The Limbic-Reticular Coupling Theory of Memory Processing in the Brain and Its Greater Compatibility over Other Theories. Dementia \& Neuropsychologia, 12, 105-113. https://doi.org/10.1590/1980-57642018dn12-020002

[28] Newman, E.L., Gillet, S.N., Climer, J.R. and Hasselmo, M.E. (2013) Cholinergic Blockade Reduces Theta-Gamma Phase Amplitude Coupling and Speed Modulation of Theta Frequency Consistent with Behavioral Effects on Encoding. Journal of Neuroscience, 33, 19635-19646. https://doi.org/10.1523/JNEUROSCI.2586-13.2013

[29] Burke, J.F., Sharan, A.D., Sperling, M.R., Ramayya, A.G., Evans, J.J., Healey, M.K., Beck, E.N., Davis, K.A., Lucas II, T.H. and Kahana, M.J. (2014) Theta and High Frequency Activity Mark Spontaneous Recall of Episodic Memories. Journal of Neuroscience, 34, 11355-11365. https://doi.org/10.1523/JNEUROSCI.2654-13.2014

[30] Cape, E.G., Manns, I.D., Alonso, A., Beaudet, A. and Jones, B.E. (2000) Neurotensin-Induced Bursting of Cholinergic Basal Forebrain Neurons Promotes Gamma and Theta Cortical Activity Together with Waking and Paradoxical Sleep. The Journal of Neuroscience, 20, 8452-8461. https://doi.org/10.1523/JNEUROSCI.20-22-08452.2000

[31] Mena-Segovia, J., Sims, H.M., Magill, P.J. and Bolam, J.P. (2008) Cholinergic Brainstem Neurons Modulate Cortical Gamma Activity during Slow Oscillations. The Journal of Physiology, 586, 2947-2960. https://doi.org/10.1113/jphysiol.2008.153874

[32] Hampel, H., Mesulam, M.M., Cuello, A.C., Farlow, M.R., Giacobini, E., Grossberg, G.T., Khachaturian, A.S., Vergallo, A., Cavedo, E., Snyder, P.J. and Khachaturian, Z.S. (2018) The Cholinergic System in the Pathophysiology and Treatment of Alzheimer's Disease. Brain, 141, 1917-1933. https://doi.org/10.1093/brain/awy132 